\title{
Respiratory Care Received by Individuals With Duchenne Muscular Dystrophy From 2000 to 2011
}

\author{
Jennifer G Andrews PhD, Aida Soim PhD, Shree Pandya PT DPT MSc, \\ Christina P Westfield RN BSN, Emma Ciafaloni MD, Deborah J Fox MPH, \\ David J Birnkrant MD, Christopher M Cunniff MD, Daniel W Sheehan PhD MD, and the \\ Muscular Dystrophy Surveillance, Tracking, and Research Network (MD STARnet)
}

\begin{abstract}
BACKGROUND: Duchenne muscular dystrophy (DMD) causes progressive respiratory muscle weakness and decline in function, which can go undetected without monitoring. DMD respiratory care guidelines recommend scheduled respiratory assessments and use of respiratory assist devices. To determine the extent of adherence to these guidelines, we evaluated respiratory assessments and interventions among males with DMD in the Muscular Dystrophy Surveillance, Tracking, and Research Network (MD STARnet) from 2000 to 2011. METHODS: MD STARnet is a populationbased surveillance system that identifies all individuals born during or after 1982 residing in Arizona, Colorado, Georgia, Hawaii, Iowa, and western New York with Duchenne or Becker muscular dystrophy. We analyzed MD STARnet respiratory care data for non-ambulatory adolescent males (12-17 y old) and men ( $\geq 18$ y old) with DMD, assessing whether: (1) pulmonary function was measured twice yearly; (2) awake and asleep hypoventilation testing was performed at least yearly; (3) home mechanical insufflation-exsufflation, noninvasive ventilation, and tracheostomy/ventilators were prescribed; and (4) pulmonologists provided evaluations. RESULTS: During 2000-2010, no more than 50\% of both adolescents and men had their pulmonary function monitored twice yearly in any of the years; $67 \%$ or fewer were assessed for awake and sleep hypoventilation yearly. Although the use of mechanical insufflation-exsufflation and noninvasive ventilation is probably increasing, prior use of these devices did not prevent all tracheostomies, and at least 18 of 29 tracheostomies were performed due to acute respiratory illnesses. Fewer than $32 \%$ of adolescents and men had pulmonologist evaluations in 2010-2011. CONCLUSIONS: Since the 2004 publication of American Thoracic Society guidelines, there have been few changes in pulmonary clinical practice. Frequencies of respiratory assessments and assist device use among males with DMD were lower than recommended in clinical guidelines. Collaboration of respiratory therapists and pulmonologists with clinicians caring for individuals with DMD should be encouraged to ensure access to the full spectrum of in-patient and out-patient pulmonary interventions. Key words: Duchenne muscular dystrophy; neuromuscular diseases; noninvasive ventilation; hypoventilation; tracheostomy; respiratory care; clinical guidelines. [Respir Care 2016;61(10):1349-1359. (C) 2016 Daedalus Enterprises]
\end{abstract}

\section{Introduction}

Duchenne muscular dystrophy (DMD) is an X-linked progressive neuromuscular disorder with an overall United

Dr Andrews is affiliated with the Department of Pediatrics, University of Arizona College of Medicine, Tucson, Arizona. Dr Soim, Ms Westfield, and Ms Fox are affiliated with the New York State Department of Health,
States prevalence in males of 1.02 per $10,000 .{ }^{1}$ DMD involves progressive weakness of the skeletal, cardiac, and respiratory muscles with pulmonary issues as a leading

Albany, New York. Dr Pandya and Dr Ciafaloni are affiliated with the Department of Neurology, University of Rochester, Rochester, New York. Dr Birnkrant is affiliated with the Department of Pediatrics, MetroHealth Medical Center, Cleveland, Ohio. Dr Cunniff is affiliated with the Department of Pediatrics, Weill Cornell Medical College, 


\section{Respiratory CARe Received IN DMD}

cause of mortality. Progressive respiratory weakness results in an ineffective cough, recurrent pneumonias, hypoventilation with sleep, and eventually daytime respiratory insufficiency. ${ }^{2-4}$ Respiratory muscles often weaken "silently" without dyspnea on exertion because loss of ambulation occurs before a clinically important decline in pulmonary function. ${ }^{5}$ Consequently, loss of respiratory muscle reserve might not be recognized by the individual with DMD, his family, or his health-care providers until an acute respiratory event occurs, leading to hospitalization or even death. Thus, proactive monitoring of pulmonary function is essential for the prevention and management of chronic respiratory insufficiency.

Modern respiratory management strategies have been shown to improve life expectancy and quality of life for individuals with DMD..$^{2,6-9}$ The use of manual and mechanical cough assistance to promote effective airway clearance is critical to the prevention of pneumonia. ${ }^{6,7,10}$ When combined with noninvasive lung ventilation, these interventions can significantly reduce morbidity and delay mortality associated with respiratory compromise. ${ }^{8,11-15}$

With the evolution of best practice respiratory strategies, several key consensus documents have been published to aid clinicians in respiratory assessment and management of individuals with DMD.4,16-18 In 2004, the American Thoracic Society (ATS) published a consensus statement on respiratory care in patients with DMD. ${ }^{4}$ More recently in 2010, the Centers for Disease Control and Prevention (CDC) sponsored and published DMD Care Considerations. ${ }^{17,18}$ Both of these consensus statements recommend twice yearly respiratory assessments after individuals lose ambulation, but the CDC-sponsored DMD Care Considerations are more specific with respect to threshold FVC values that trigger evaluations for sleep and awake hypoventilation and initiation of noninvasive respiratory device use.

Cornell University, New York, New York. Dr Sheehan is affiliated with the Department of Pediatrics, University at Buffalo, State University of New York, Buffalo, New York.

Supplementary material related to this paper is available at http:// www.rcjournal.com.

The Muscular Dystrophy Surveillance, Tracking, and Research Network (MD STARnet) is supported by Centers for Disease Control and Prevention cooperative agreements 5U01DD000191-04, 5U01DD000190-04, 5U01DD000187-04, and 5U01DD000189-04. Dr Birnkrant has disclosed a relationship with Hill-Rom Corporation. No other authors have disclosed any conflicts of interest.

Correspondence: Daniel W Sheehan PhD MD, Lung Center, Women and Children's Hospital of Buffalo, 219 Bryant Street, Buffalo, NY 14222. E-mail: dsheehan@upa.chob.edu.

DOI: $10.4187 /$ respcare.04676

\section{QUICK LOOK}

\section{Current knowledge}

The use of noninvasive ventilation and mechanical cough assist decreases morbidity and prolongs survival in Duchenne muscular dystrophy (DMD). Without respiratory function monitoring, progressive respiratory muscle weakness can go undetected. Respiratory care guidelines for DMD recommend scheduled respiratory assessments to proactively determine the need for respiratory assist devices.

\section{What this paper contributes to our knowledge}

Between 2000 and 2010, in a large sample of adolescents and adults with DMD in the United States, monitoring of respiratory function for weak coughing and awake and sleep hypoventilation occurred less often than recommended by the 2004 American Thoracic Society guidelines. The use of noninvasive respiratory assist devices was lower than recommended as well.

Despite these consensus statements, respiratory complications continue to be a common cause of morbidity and mortality in individuals with DMD. Based on communications with DMD advocacy groups, we hypothesized that adherence to guidelines would be variable across the different states and less than recommended. In this study, we examined the respiratory assessments and treatment of individuals in the Muscular Dystrophy Surveillance, Tracking, and Research Network (MD STARnet) between 2000 and 2011. The purpose of this study was 2-fold: first, to describe the respiratory management of the individuals in our multi-site network, and second, to compare that management with contemporary guidelines that seek to improve the respiratory care of individuals with DMD and provide early detection and proactive management of respiratory complications.

\section{Methods}

\section{Subjects and Study Design}

MD STARnet is the largest population-based surveillance system of individuals with Duchenne and Becker muscular dystrophy in the United States funded by the CDC since 2002. MD STARnet includes data on individuals with Duchenne or Becker Muscular dystrophy who were (1) born between January 1982 and October 2011; (2) diagnosed by age $21 \mathrm{y}$; and (3) resided at any point in time in Arizona, Colorado, Georgia, Hawaii, Iowa, or 12 counties in western New York. Arizona, Colorado, Iowa, 


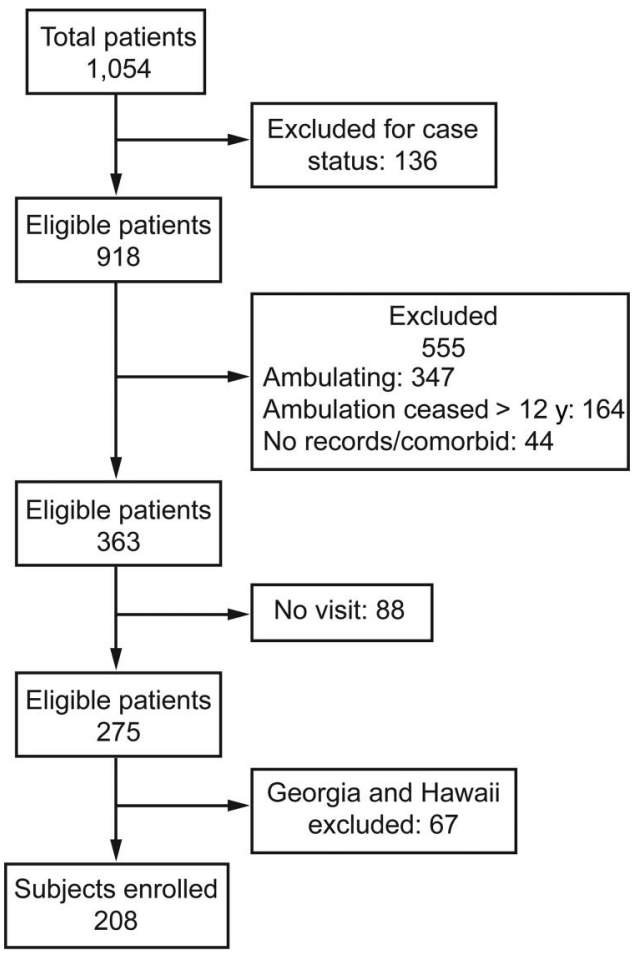

Fig. 1. Flow chart.

and western New York started data collection in 2004, Georgia started in 2005, and Hawaii started in 2008. Individuals identified before September 2011 were followed through December 2011, whereas individuals identified between September 2011 and December 2011 were followed through December 2012. Data were collected through medical record abstraction of in-patient and out-patient records from multiple hospital and clinic locations within each state, and each case was classified by a systematic clinician review process with a ranking of definite, probable, possible, asymptomatic for males with DMD, or an affected female with DMD using a rigorous algorithm and consensus process that has been described previously. ${ }^{19,20}$ For each case, abstraction was performed once yearly within a year; therefore, 2010 was the last year with complete whole year data. Figure 1 demonstrates the inclusion criteria used for this study, resulting in an analyzable sample of 208 individuals, $11 \%$ of which were siblings. We excluded Hawaii and Georgia cases due to restricted case ascertainment and incomplete access to pulmonary sources, respectively. We also excluded individuals with Becker muscular dystrophy from analysis. Given the less severe respiratory course of Becker muscular dystrophy and since guidelines are specific to DMD, we excluded individuals with Becker from analysis using ambulation beyond $12 \mathrm{y}$ of age as a clinical diagnostic differentiator. Individuals with neurologic co-occurring conditions that would affect ambulation status were also excluded from the study to prevent any inclusion of Becker patients. Asymptomatic cases were excluded due to the absence of clinical symptomatology, and possible cases were excluded for their lack of confirmatory diagnostic data. Last, females were excluded from analysis due to their unique clinical course distinct from that of males with DMD. ${ }^{21}$ This study further focused on a subset of these individuals who were classified as either definite or probable DMD, were $\geq 12$ y of age between 2000 and 2010, with documentation of at least one clinic visit with any type of provider in any year between 2000 and 2010. From those, we identified individuals as having DMD by selecting those who had lost ambulation by age $12 \mathrm{y}$. Each of the MD STARnet sites included in the analysis has a combination of pediatric, adult, and combined locations for neuromuscular and pulmonary care for the population. Most of the clinics did not have pulmonary care incorporated into the neuromuscular clinics at the time of data collection. All MD STARnet sites obtained institutional review board approvals or had public health authority permitting collection of these data.

\section{Measures}

Using surveillance data collected for visits that occurred between January 1, 2001 and December 31, 2010, we examined a wide range of respiratory assessments, evaluations, and interventions to describe respiratory management and adherence to established respiratory care guidelines. Cases were grouped according to their age during each visit year as either adolescents (12-17 y old) or adults ( $\geq 18$ y old). These age groups were chosen to document any variations in care received by adolescents and adults with DMD. Adolescents with DMD typically receive care from pediatric providers, whereas adults with DMD may receive care from adult or pediatric providers, depending on availability of providers trained in neuromuscular respiratory care. Individuals were classified as having used steroids if they had any steroid use documented.

Qualified respiratory assessments included FVC, cough peak flow, maximum expiratory pressure $\left(\mathrm{P}_{\mathrm{Emax}}\right), \mathrm{S}_{\mathrm{pO}_{2}}$, end-tidal $\mathrm{P}_{\mathrm{CO}_{2}}\left(\mathrm{P}_{\mathrm{ETCO}_{2}}\right)$, and either arterial, venous, or capillary blood $\mathrm{P}_{\mathrm{CO}_{2}}$ for each test that was documented as performed. Qualified respiratory evaluations include recommendation or completion of a sleep evaluation (polysomnogram, overnight oximetry, or sleep study not otherwise specified). Individuals were considered to have used respiratory assistive devices, including a home mechanical in-exsufflator or home nocturnal noninvasive ventilation (NIV) if the device was prescribed to the individual. Assisted ventilation via tracheostomy was also included. For individuals who used a tracheostomy, we established the year and age at which tracheostomy was performed; 
Table 1. Denominator Values Used in Creating Proportions in Tables 2 and 3

\begin{tabular}{|c|c|c|c|c|c|c|c|c|c|c|c|c|}
\hline & Age, y & 2000 & 2001 & 2002 & 2003 & 2004 & 2005 & 2006 & 2007 & 2008 & 2009 & 2010 \\
\hline \multirow[t]{2}{*}{ Denominator $1^{*}$} & $12-17$ & 57 & 71 & 74 & 71 & 75 & 82 & 83 & 71 & 70 & 67 & 74 \\
\hline & $\geq 18$ & 1 & 4 & 6 & 17 & 17 & 21 & 29 & 39 & 50 & 50 & 61 \\
\hline \multirow[t]{2}{*}{ Denominator $2 \dagger$} & $12-17$ & 55 & 65 & 66 & 68 & 72 & 78 & 80 & 81 & 68 & 67 & 72 \\
\hline & $\geq 18$ & 0 & 3 & 4 & 10 & 9 & 14 & 20 & 27 & 39 & 38 & 45 \\
\hline \multirow[t]{2}{*}{ Denominator $3 \ddagger$} & $12-17$ & 54 & 64 & 63 & 65 & 66 & 68 & 67 & 58 & 46 & 40 & 42 \\
\hline & $\geq 18$ & 0 & 3 & 2 & 7 & 7 & 10 & 12 & 18 & 15 & 8 & 10 \\
\hline \multirow[t]{2}{*}{ Denominator Sleep§ } & $12-17$ & 48 & 54 & 48 & 46 & 48 & 48 & 53 & 56 & 46 & 42 & 44 \\
\hline & $\geq 18$ & 0 & 2 & 3 & 3 & 4 & 6 & 7 & 7 & 12 & 8 & 6 \\
\hline \multirow[t]{2}{*}{ Denominator MI/E\|| } & $12-17$ & 1 & 3 & 3 & 6 & 5 & 8 & 13 & 17 & 14 & 21 & 27 \\
\hline & $\geq 18$ & 0 & 0 & 0 & 1 & 0 & 2 & 1 & 3 & 7 & 9 & 14 \\
\hline
\end{tabular}

\footnotetext{
* Denominator 1 includes a count of all cases by year for which a physician visit is documented excluding year of death.

$\dagger$ Denominator 2 includes all cases in denominator 1 less any documented visits that occurred after tracheostomy was performed.

‡ Denominator 3 includes all cases in denominator 2 less any documented visits occurring after mechanical in-exsufflation was recommended or prescribed.

$\S$ Sleep denominator includes all cases in denominator 1 less any documented visits that occurred after noninvasive or invasive ventilation was prescribed.

$\|$ Denominator 1 excluding all visits occurring before first year when cough peak flow or PEmax fell below benchmarks per 2004 ATS Consensus Statement.

$\mathrm{MI} / \mathrm{E}=$ mechanical in-exsufflation .
}

whether tracheostomies were performed due to an acute respiratory illness, electively (not acutely ill but with deteriorating respiratory muscle function), or unknown; whether respiratory assessments were performed in the year before the tracheostomy; and whether home mechanical in-exsufflation or NIV (including mask/nasal and sip ventilation) were used in the year before tracheostomy placement.

\section{Statistical Analysis}

Descriptive statistics were used to report frequencies, means, and ranges. All data were analyzed using SAS 9.2 (Cary, North Carolina). Full data were evaluated across all years, and site-specific differences or variations in practice were reviewed for the 2010 study year to determine the presence of any site-specific bias using chi-square analysis. To evaluate sample representativeness, $t$ test and chisquare tests were used to assess differences between those eligible for the analytic sample and the total MD STARnet population. Linear regression was used to analyze the change over time in the age at which tracheostomy was performed. A $P$ value of $<.05$ was considered significant. Numeric values used in the denominators can be found in the supplemental tables (see the supplementary materials at http://www.rcjournal.com).

\section{Results}

\section{Participants}

Denominator data for each pulmonary function measurement assessed for the included 208 males with DMD are found in Table 1. The final sample included individ- uals from Arizona $(n=83)$, Colorado $(n=63)$, Iowa $(n=$ $40)$, and western New York $(n=22)$. Fifty-four percent of males were non-Hispanic white, 34\% Hispanic, 3\% African-American, and 9\% other (including Asian, Hawaiian or Pacific Islander, Native American, other, or multiple) or unknown. Fifty-two percent of males used corticosteroids at any time during their lifetime (data not shown). There was a difference in the distribution of cases from each site, with New York contributing fewer cases than expected; however, a large majority of individuals in western New York were prescribed corticosteroids early (67\%), which may have extended ambulation for cases who had DMD beyond age 12 y (33\%), thus excluding them as if they had Becker muscular dystrophy based on the use of age at which ambulation ceased as a proxy (data not shown).22,23 There were also more Hispanics in the analytic data set than in the MD STARnet population overall. Total $n$ for adolescents and adults in each year is available in supplemental Table 1.

In 2000 , the average age \pm SD of our sample was $9 \pm 4$ y (data not shown). On average, the sample ceased ambulation at $10 \pm 1 \mathrm{y}$ of age and initiated nocturnal NIV and mechanical in-exsufflation at $16 \pm 3$ and $17 \pm 3$ y of age, respectively. The age of initiation of noninvasive devices varied greatly from $<8$ to $>25$ y. Tracheostomies were performed between 2000 and 2010 on individuals as young as $15 \mathrm{y}$ of age in our sample (Fig. 2).

\section{Respiratory Assessments}

For wheelchair users with DMD or $\geq 12$ y old, the 2004 ATS consensus statement recommends visits with a "physician specializing in pediatric respiratory care" and respi- 


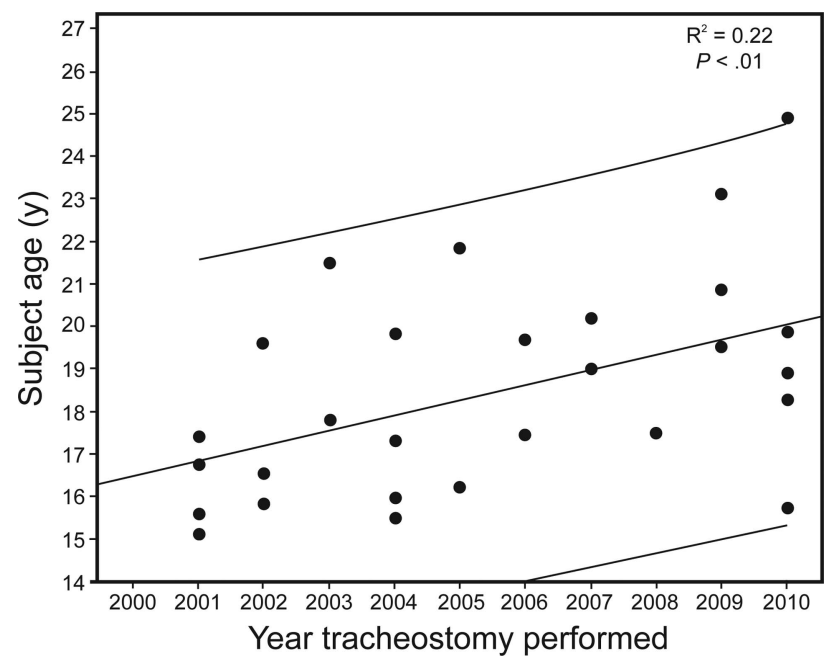

Fig. 2. Age in years when tracheostomy was performed and year of tracheostomy for subjects with Duchenne muscular dystrophy.

ratory assessments (FVC, cough peak flow, $\mathrm{P}_{\mathrm{Emax}}$, and $\mathrm{S}_{\mathrm{pO}_{2}}$ ) a minimum of once every 6 months or annually for $\mathrm{P}_{\mathrm{ETCO}}$ or blood $\mathrm{P}_{\mathrm{CO}_{2}}$, which includes either arterial, venous, or capillary measurements., 47,18

FVC Monitoring. For all sites combined, FVC measurements were completed annually for $65-85 \%$ of adolescents between 2000 and 2010 and 33-100\% of adults between 2000 and 2010 , but $\leq 50 \%$ of adolescents and $\leq 41 \%$ of adults received $\geq 2 \mathrm{FVC}$ measurements/y during these same times (Fig. 3). Moreover, there has been no consistent increase in the proportion of individuals receiving twice yearly FVC monitoring over time since the release of the ATS recommendations in 2004 (Table 2). In 2010, the average frequency of monitoring across the 4 individual MD STARnet sites ranged for all individuals combined from 20 to $69 \%$ for annual FVC monitoring and from 20 to $55 \%$ for semiannual FVC monitoring with significant in differences in at least annual measurement across sites $(P=.001)$.

Cough Peak Flow and $\mathbf{P}_{\text {Emax }}$ Monitoring. For all sites combined, cough peak flow or $\mathrm{P}_{\text {Emax }}$ assessments were rarely completed, with $0 \%$ monitoring for most years (Table 2). When completed, $2-12 \%$ of adolescents and $7-30 \%$ of adults had cough peak flow or $\mathrm{P}_{\mathrm{Emax}}$ assessments annually. Similarly, $<5 \%$ of adolescents and $<20 \%$ of adults had semiannual assessments during the years when assessments were completed (Table 2). In 2010, for the 4 individual sites of MD STARnet, cough peak flow and $\mathrm{P}_{\mathrm{Emax}}$ monitoring at least twice yearly ranged from 0 to $20 \%$, with significant differences in at least annual measurement across sites $(P=.001$ and $P=.005$, respectively).
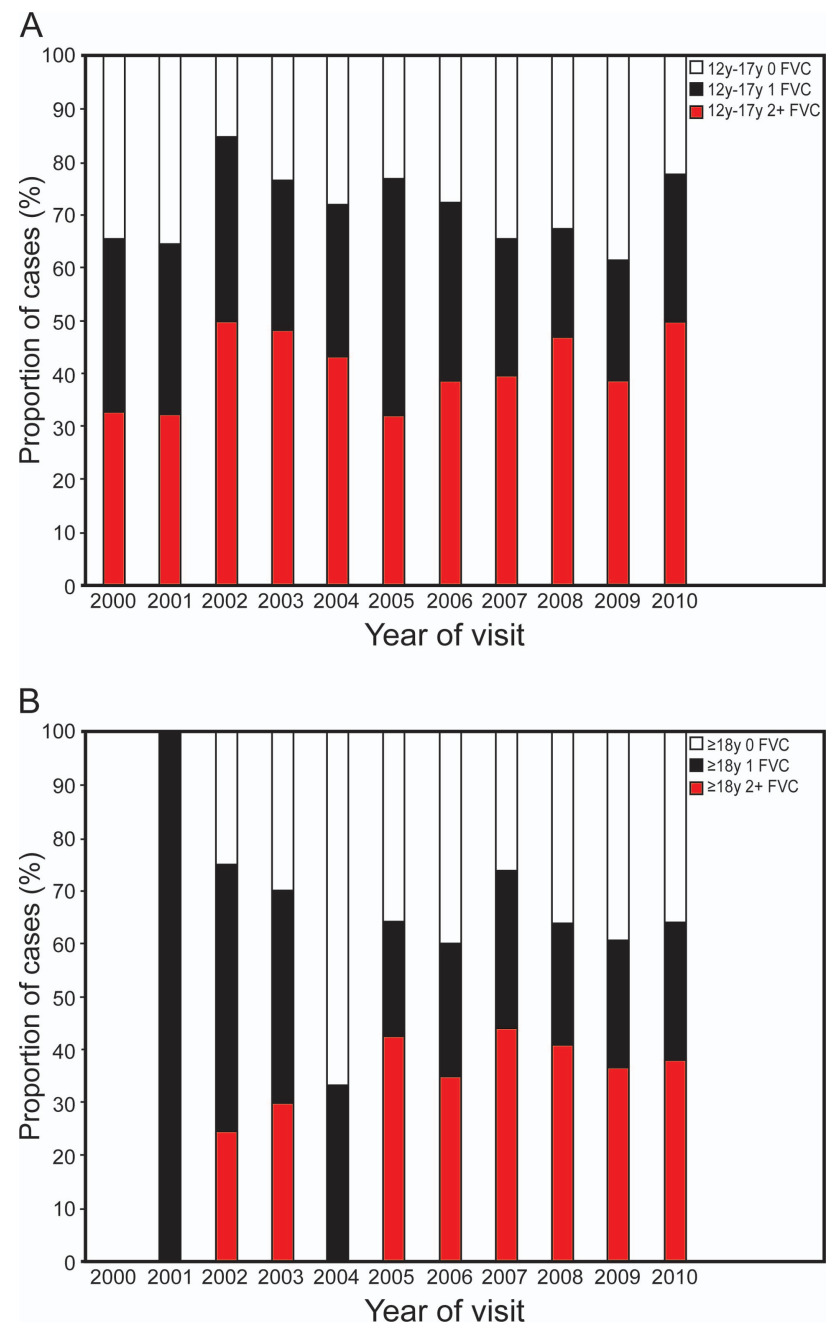

Fig. 3. A: FVC monitoring frequency in non-ambulatory adolescents (12-17 y old) with Duchenne muscular dystrophy. B: FVC monitoring frequency in non-ambulatory adults ( $\geq 18 \mathrm{y}$ old) with Duchenne muscular dystrophy.

$\mathbf{S}_{\mathbf{p O}_{2}}$. The frequency of any $S_{\mathrm{pO}_{2}}$ completed assessment increased from 14 to $41 \%$ across all years for adolescents and varied from 0 to $67 \%$ for adults during years when an assessment was completed. Completion of semiannual $\mathrm{S}_{\mathrm{pO}_{2}}$ assessments ranged from 2 to $14 \%$ for adolescents; greater variability was found for adults, with $0-33 \%$ having completed assessments semiannually (Table 2). In 2010, for the 4 individual sites of MD STARnet, the frequency of semiannual monitoring ranged from 9 to $36 \%$ for $\mathrm{S}_{\mathrm{pO}_{2}}$, with significant differences in annual measurement across sites $(P=.001)$.

Awake $\mathbf{P}_{\mathbf{C O}_{2}}$ Monitoring. Annual measures of $\mathrm{P}_{\mathrm{ETCO}_{2}}$ or blood $\mathrm{P}_{\mathrm{CO}_{2}}$ occurred in $\leq 27 \%$ of adolescents monitored and $\leq 18 \%$ of adults (Table 2). In 2010, for the 4 individual sites of MD STARnet, annual monitoring of $\mathrm{P}_{\mathrm{ETCO}_{2}}$ or blood $\mathrm{P}_{\mathrm{CO}_{2}}$ ranged from 5 to $50 \%$ of individuals, with 
Table 2. Frequency of Respiratory Assessments for Non-Ambulatory Adolescents (12-17 Years Old) and Men ( $\geq 18$ Years Old) From MD STARnet 2000-2010

\begin{tabular}{|c|c|c|c|c|c|c|c|c|}
\hline \multirow{2}{*}{ Assessment Type } & \multirow{2}{*}{$\begin{array}{l}\text { Assessment } \\
\text { Frequency }\end{array}$} & \multirow{2}{*}{ Age, $y$} & \multicolumn{6}{|c|}{$\%$ With Assessment Frequency } \\
\hline & & & 2000 & 2002 & 2004 & 2006 & 2008 & 2010 \\
\hline \multirow[t]{4}{*}{$\mathrm{FVC}^{*}$} & $\geq 2$ & $12-17$ & 33 & 50 & 43 & 39 & 47 & 50 \\
\hline & & $\geq 18$ & N/A & 25 & 0 & 35 & 41 & 38 \\
\hline & $\geq 1$ & $12-17$ & 65 & 85 & 72 & 73 & 68 & 78 \\
\hline & & $\geq 18$ & N/A & 75 & 33 & 60 & 64 & 65 \\
\hline \multirow[t]{4}{*}{ Cough peak flow $\dagger$} & $\geq 2$ & $12-17$ & 0 & 0 & 0 & 0 & 4 & 3 \\
\hline & & $\geq 18$ & N/A & 0 & 0 & 0 & 0 & 20 \\
\hline & $\geq 1$ & $12-17$ & 0 & 0 & 0 & 6 & 6 & 12 \\
\hline & & $\geq 18$ & N/A & 0 & 0 & 0 & 7 & 20 \\
\hline \multirow[t]{4}{*}{$\mathrm{P}_{\text {Emax }} \dagger$} & $\geq 2$ & $12-17$ & 2 & 0 & 0 & 0 & 4 & 3 \\
\hline & & $\geq 18$ & N/A & 0 & 0 & 0 & 0 & 20 \\
\hline & $\geq 1$ & $12-17$ & 2 & 2 & 0 & 5 & 7 & 12 \\
\hline & & $\geq 18$ & N/A & 0 & 0 & 0 & 7 & 30 \\
\hline \multirow{4}{*}{$\mathrm{S}_{\mathrm{pO}_{2}} \ddagger$} & $\geq 2$ & $12-17$ & 2 & 7 & 9 & 7 & 11 & 14 \\
\hline & & $\geq 18$ & 0 & 33 & 6 & 21 & 14 & 12 \\
\hline & $\geq 1$ & $12-17$ & 14 & 15 & 20 & 22 & 33 & 41 \\
\hline & & $\geq 18$ & 0 & 67 & 35 & 45 & 38 & 38 \\
\hline \multirow[t]{2}{*}{$\mathrm{P}_{\mathrm{ETCO}_{2}}$ or blood $\mathrm{P}_{\mathrm{CO}_{2}} \ddagger$} & $\geq 1$ & $12-17$ & 4 & 8 & 5 & 7 & 13 & 27 \\
\hline & & $\geq 18$ & 0 & 17 & 18 & 10 & 8 & 16 \\
\hline \multirow{2}{*}{$\begin{array}{l}\text { Sleep evaluation (polysomnogram, overnight } \\
\text { oximetry, or not otherwise specified)§ }\end{array}$} & $\geq 1$ & $12-17$ & 4 & 6 & 6 & 13 & 15 & 23 \\
\hline & & $\geq 18$ & N/A & 33 & 0 & 2 & 17 & 33 \\
\hline \multicolumn{9}{|c|}{$\begin{array}{l}\text { Odd years are excluded for readability. } \\
\text { * Denominator } 1 \text { includes a count of all cases by year for which a physician visit is documented excluding year of death. } \\
\dagger \text { Denominator } 2 \text { includes all cases in denominator } 1 \text { less any documented visits that occurred after tracheostomy was performed. } \\
\text { † Denominator } 3 \text { includes all cases in denominator } 2 \text { less any documented visits occurring after mechanical in-exsufflation was recommended or prescribed. } \\
\S \text { Sleep denominator includes all cases in denominator } 2 \text { less any documented visits occurring after nocturnal noninvasive ventilation was recommended or prescribed. } \\
\text { N/A = not applicable; no cases present in the denominator }\end{array}$} \\
\hline
\end{tabular}

significant differences in at least annual measurement across sites $(P=.01)$.

Sleep Evaluations. Sleep hypoventilation monitoring through sleep evaluations is recommended annually for individuals with DMD who are in a wheelchair. ${ }^{4}$ We evaluated the proportion of individuals without tracheostomy and before recommendation of nocturnal NIV use who received annual sleep studies. Non-ambulatory adolescents with an annual sleep evaluation ranged from approximately 4\% in 2000 to $23 \%$ in 2010 (Table 2). Among non-ambulatory adults, sleep evaluations were variably completed, fluctuating between 0 and up to $33 \%$. In 2010, for the 4 individual sites of MD STARnet, sleep evaluation at least once yearly ranged from 0 to $80 \%$, with significant differences in at least annual measurement across sites $(P=.01)$.

\section{Respiratory Interventions}

Mechanical in-exsufflator use is recommended when cough peak flow falls below $270 \mathrm{~L} / \mathrm{min}$ or $\mathrm{P}_{\text {Emax }}$ falls below $60 \mathrm{~mm} \mathrm{Hg}$ per ATS guidelines. ${ }^{4}$ The placement of a tracheostomy under ATS guidelines indicates medical necessity or failure of NIV due to nonadherence, severe weakness, or the lack of infrastructure to support noninvasive interventions.

Noninvasive Respiratory Care Device Use (Mechanical In-Exsufflator; NIV). Mechanical in-exsufflator use among all adolescents increased from $0 \%$ in 2000 to $31 \%$ in 2010 and from $0 \%$ in 2000 to $74 \%$ in 2010 among all adults (Table 3). Mechanical in-exsufflator use for those individuals with documented reduced cough peak flow or $\mathrm{P}_{\text {Emax }}$ values ranged from 46 and $100 \%$ in 2006 to 63 and $71 \%$ in 2010 for adolescents and adults, respectively (Table 3). Thresholds for initiating nocturnal NIV are not discussed in the ATS guidelines. Nocturnal NIV use ranged from 7 to $27 \%$ across all years for all adolescents and from 56 to $66 \%$ for adults from 2004 to 2010 (Table 3). In 2010, for the 4 individual sites of MD STARnet, individuals with mechanical in-exsufflation ranged from 42 to $62 \%$, with no significant differences across sites $(P=.77)$ and from 33 to $100 \%$ for individuals with reduced cough peak flow 
Table 3. Percentages of Non-Ambulatory Adolescents (12-17 Years Old) and Men ( $\geq 18$ Years Old) With Noninvasive Respiratory Care Use From MD STARnet 2000-2010

\begin{tabular}{|c|c|c|c|c|c|c|c|}
\hline \multirow{2}{*}{ Respiratory Care } & \multirow{2}{*}{ Age, y } & \multicolumn{6}{|c|}{$\%$ With NIV Use in Year } \\
\hline & & 2000 & 2002 & 2004 & 2006 & 2008 & 2010 \\
\hline \multicolumn{8}{|l|}{$\mathrm{MI} / \mathrm{E}$} \\
\hline \multirow[t]{2}{*}{ All cases* } & $12-17$ & 0 & 3 & 3 & 12 & 26 & 31 \\
\hline & $\geq 18$ & 0 & 17 & 29 & 38 & 46 & 74 \\
\hline \multirow[t]{2}{*}{ After $\mathrm{PCF}<270 \mathrm{~L} / \mathrm{min}$ or $\mathrm{P}_{\mathrm{Emax}}<60 \mathrm{~mm} \mathrm{Hg} \dagger$} & $12-17$ & N/A & N/A & N/A & 46 & 57 & 63 \\
\hline & $\geq 18$ & N/A & N/A & N/A & 100 & 57 & 71 \\
\hline \multirow[t]{2}{*}{ Nocturnal NIV $\dagger$} & $12-17$ & 7 & 14 & 21 & 24 & 20 & 27 \\
\hline & $\geq 18$ & 0 & 17 & 65 & 66 & 56 & 63 \\
\hline \multicolumn{8}{|c|}{$\begin{array}{l}\text { Odd years are excluded for readability. } \\
\text { * Denominator } 1 \text { includes a count of all cases by year for which a physician visit is documented excluding year of death. } \\
\dagger \text { Denominator } 1 \text { excludes all visits occurring before the first year when cough peak flow or maximum expiratory pressure fell below benchmarks per } 2004 \text { ATS Consensus Statement. } \\
\text { N/A = not applicable; no cases present in the denominator } \\
\text { MI/E = mechanical in-exsufflation } \\
\text { PCF = cough peak flow }\end{array}$} \\
\hline
\end{tabular}

Table 4. Tracheostomies Performed in 2 Year Periods From MD STARnet 2001-2010

\begin{tabular}{|c|c|c|c|c|c|c|c|c|c|}
\hline \multirow{3}{*}{ Years } & \multirow{3}{*}{$\begin{array}{l}\text { No. of New } \\
\text { Tracheostomies }\end{array}$} & \multirow{3}{*}{$\begin{array}{l}\text { Median Age at } \\
\text { New Tracheostomy } \\
\text { (Range) }\end{array}$} & \multirow{3}{*}{$\begin{array}{c}\% \text { of } \\
\text { Tracheostomies } \\
\text { Due to Acute Illness* }\end{array}$} & \multicolumn{6}{|c|}{ In the Year Before Tracheostomy, \% of Individuals With: } \\
\hline & & & & \multirow{2}{*}{$\begin{array}{l}\text { Clinic } \\
\text { Visit }\end{array}$} & \multirow{2}{*}{$\mathrm{MI} / \mathrm{E}$} & \multirow[t]{2}{*}{ NIV } & \multirow[t]{2}{*}{ FVC } & \multicolumn{2}{|c|}{$\begin{array}{c}\text { Assessment for Daytime } \\
\text { Hypoventilation }\end{array}$} \\
\hline & & & & & & & & $\mathrm{S}_{\mathrm{O}_{2}}$ & $\mathrm{P}_{\mathrm{ETCO}_{2}}$ or $\mathrm{P}_{\mathrm{aCO}_{2}}$ \\
\hline 2001-2002 & 7 & $16.5(15.1-19.6)$ & 71 & 86 & 0 & 29 & 57 & 43 & 14 \\
\hline 2003-2004 & 6 & $17.5(15.5-21.4)$ & 33 & 83 & 17 & 67 & 67 & 50 & 33 \\
\hline 2005-2006 & 4 & $17.4(16.2-21.8)$ & 100 & 100 & 25 & 50 & 50 & 25 & 0 \\
\hline $2007-2008$ & 4 & $18.2(17.4-20.2)$ & 100 & 100 & 50 & 50 & 75 & 0 & 0 \\
\hline 2009-2010 & 8 & $19.7(15.7-24.9)$ & 13 & 100 & 100 & 88 & 63 & 38 & 13 \\
\hline $\begin{array}{l}\text { * Estimated fron } \\
\mathrm{MI} / \mathrm{E}=\text { mechani } \\
\mathrm{NIV}=\text { noninvas } \\
\mathrm{P}_{\mathrm{ETCO}_{2}=\text { end-ti }}\end{array}$ & $\begin{array}{l}\text { ospital records percenta } \\
\text { in-exsufflation } \\
\text { ventilation } \\
\text { carbon dioxide pressur }\end{array}$ & is "at least." & & & & & & & \\
\hline
\end{tabular}

or $\mathrm{P}_{\text {Emax }}$ values. Nocturnal NIV across the sites ranged from 31 to $63 \%$ in 2010 with no significant differences across sites $(P=.08)$.

Tracheostomy/Ventilator. Between January 1, 2001, and December 31, 2010, 29 tracheostomies were performed. The majority (86\%) occurred before $21 \mathrm{y}$ of age (Fig. 2). In 2-y periods between 2001 and 2010, tracheostomies performed during acute respiratory illnesses ranged from 13 to $100 \%$. In the year before tracheostomy, $\leq 50 \%$ had an evaluation for daytime hypoventilation (Table 4). In 2009-2010, 100\% had mechanical in-exsufflation and $88 \%$ had NIV before tracheostomy (Table 4 ). The age at which tracheostomy is performed has increased significantly from approximately 16 to $19 \mathrm{y}$ of age between 2001 and 2010 (Fig. 2). There were 8 tracheostomies performed in 2009-2010; one site had only one individual receive a tracheostomy at age $16 \mathrm{y}$, and the re- maining sites had 2 or 3 individuals receive tracheostomies, and the mean age of performance was between 20 and 22 y (data not shown).

\section{Pulmonologist Involvement in Care}

Pulmonologist visits were abstracted annually; however, only the date of the most recent visit was retained. For example, if an individual with DMD was seen by a pulmonologist in 2011, we could not determine whether he was also seen in 2010. As a result, we examined pulmonology visits from January 1, 2010 through August 31, 2011 during which time only $41 \%$ of adolescents and $26 \%$ of men were seen by a pulmonologist. Among individual MD STARnet sites, between 35 and 50\% of all non-ambulatory adolescents and $20-53 \%$ of non-ambulatory adults were evaluated by a pulmonologist during this time. 


\section{Duchenne Muscular Dystrophy Consensus Guidelines 2010}

These findings provide baseline data for future studies evaluating the impact of and compliance with the 2010 CDC-sponsored DMD Care Considerations. ${ }^{17,18}$ The CDC Care Considerations do not differ greatly from the ATS consensus statement and are in alignment with ATS regarding all of the previously discussed respiratory assessments. The CDC Care Considerations, however, do differ slightly from the ATS consensus statement in initiation of nocturnal NIV. Specifically, the CDC considers initiation of nocturnal NIV when there are signs of hypoventilation as measured by $\mathrm{FVC}<30 \%$ predicted, baseline $\mathrm{S}_{\mathrm{pO}_{2}}$ is $<95 \%$, and/or awake blood $\mathrm{P}_{\mathrm{CO}_{2}}$ or end-tidal $\mathrm{P}_{\mathrm{ETCO}_{2}}$ is $>45 \mathrm{~mm} \mathrm{Hg}$. For those individuals showing signs of hypoventilation as defined by the CDC, $<60 \%$ of adolescents and $<82 \%$ of adults used nocturnal NIV in any given year. In 2010, the proportion of individuals with evidence of hypoventilation and nocturnal NIV ranged from 58 to $70 \%$ across the 4 MD STARnet sites (data not shown).

\section{Discussion}

This study describes respiratory assessments and interventions provided within a United States DMD population between 2000 and 2010. We found that monitoring of respiratory function and use of respiratory assist devices occurred less often than recommended. Overall, $\leq 50 \%$ of non-ambulatory adolescents or adult men had their pulmonary function (FVC, cough peak flow, $\mathrm{P}_{\text {Emax }}$ ) monitored twice yearly, and $<67 \%$ were monitored annually for awake or asleep hypoventilation (awake $\mathrm{S}_{\mathrm{pO}_{2}}$ or blood $\mathrm{P}_{\mathrm{CO}_{2}}$, polysomnogram, overnight oximetry, or sleep study not otherwise specified). Similarly, from 2010 to 2011, $<32 \%$ of adolescents and men had a pulmonologist evaluation. In addition, between 2000 and 2010, $\geq 63 \%$ of tracheostomies were performed during acute respiratory illnesses. Although the use of mechanical in-exsufflation and NIV appears to be increasing, use of these noninvasive devices did not prevent all tracheostomies.

This study has notable strengths. This is the first population-based report concerning DMD pulmonary care and management from a surveillance system within the United States. The data provide insight into clinical practice in the United States 4 y before and 6 y after release of the 2004 ATS consensus statement. ${ }^{4}$ The data also provide a baseline for the 2010 CDC DMD Care Considerations guidelines. ${ }^{17-18}$ Finally, the use of data from a surveillance system that abstracts directly from medical record avoids limitations introduced by recall bias of care providers.

There are several limitations to our study. Our study was observational and relied on retrospective case ascertainment and data abstracted from medical records, which depends on care provider documentation. Because we did not capture some assessments that may have been recommended but were not completed (eg, sleep evaluations), we could not determine whether NIV was needed but underprescribed. We also could not determine whether subjects declined offered prescriptions for these devices. The study population differs from the MD STARnet population from which the sample was drawn. The differences in the distribution of cases across race/ethnicity and site do not directly affect the findings but may result in limitations on the generalizability of these results. Last, we could not assess actual use of NIV and mechanical in-exsufflation at home and during hospital admissions; therefore, we could not determine whether optimal use would have prevented tracheostomies.

Respiratory muscles in individuals with DMD often weaken silently, without dyspnea on exertion or other signs and symptoms. Regular monitoring of pulmonary function is essential to identify and prevent acute and chronic respiratory insufficiency. With the development of improved respiratory management strategies and other critical therapies, patients with DMD are living much longer, to the fourth decade of life and beyond, when they are monitored closely. For example, NIV has been available since the late 1980s, and since the 1990s, individuals with DMD followed at the Newcastle England Neuromuscular Centre have been offered nocturnal NIV when a home oximetry study reveals sleep hypoxemia; individuals undergo serial home oximetry studies when their regularly monitored FVC falls to $\leq 1.25$ L. In 2002, Eagle et al $^{24}$ reported that this initiation of nocturnal NIV increased the mean age of survival to $25.3 \mathrm{y}$ compared with $14.4 \mathrm{y}$ in the $1960 \mathrm{~s}$. More recently, Ishikawa et $\mathrm{al}^{9}$ reported a $50 \%$ survival to $39.6 \mathrm{y}$ when NIV and mechanically assisted cough are used. Hence, screening for respiratory muscle weakness and initiation of noninvasive respiratory aids greatly influences survival.

Our MD STARnet study found results similar to those in Canada, where the management of individuals with DMD is variable and differs from the 2004 ATS consensus statement. ${ }^{25}$ A survey of Canadian pediatric respirologists and neuromuscular specialists in 2010 found great variation in the selection of tests for evaluating pulmonary function and the timing of pulmonology consultations. To assess respiratory muscle strength, $96 \%$ of respirologists and $82 \%$ of Canadian Pediatric Neuromuscular Group members use maximum inspiratory and expiratory pressures; $82 \%$ of Canadian Pediatric Neuromuscular Group members but only $36 \%$ of respirologists use peak cough flows. Thirtyseven percent of initial consultations by respirologists occurred reactively after a patient's first admission to hospital with respiratory complications. Only a small minority of Canadian respirologists and neuromuscular specialists use mechanical in-exsufflation for out-patients, probably 


\section{Respiratory CARe Received IN DMD}

because of lack of insurance coverage for mechanical inexsufflation.

Our results regarding the frequency of respiratory assessments are also similar to those recently found for males with DMD in Europe. Rodger et $\mathrm{al}^{26}$ reported that $<50 \%$ of 10-17-y-olds and adults in the United Kingdom had respiratory assessments at least every 6 months. Across the United Kingdom, Germany, and Denmark, adults had at least annual assessment 69,75 , and $67 \%$ of the time, respectively. ${ }^{26}$ Ventilation practice also differed markedly across countries: $93 \%$ of adults with mechanical ventilation used NIV in the United Kingdom and $88 \%$ in Germany but only $24 \%$ in Denmark, with most instead using tracheostomy. ${ }^{26}$ The authors found that care for adults in the United Kingdom is considerably less comprehensive than for children, since fewer adults attend specialized neuromuscular clinics. They stressed that there is an urgent need for improved adult access to specialized clinics.

Barriers to the implementation of DMD respiratory care guidelines warrant further studies. There have been recent meta-analytic reviews of factors that contribute to low adherence to other clinical practice guidelines. Based on 12 systematic reviews that met inclusion criteria, Franke et $a^{27}$ identified factors and grouped them according to characteristics of the guidelines, implementation strategies, patients, providers, and the environment, providing a useful framework for discussing possible reasons for the low adherence rates within MD STARnet.

The recommendations from the 2004 ATS consensus statement are largely based on expert opinion or observational trials from single clinical centers and not controlled prospective studies. Thus, some providers might regard the ATS consensus statement as not evidencebased and hence not necessary to follow. Another key provider barrier that has affected the implementation of other guidelines is a knowledge barrier: lack of awareness and familiarity of health-care providers. ${ }^{28}$ Individuals with DMD and their families may also be unaware of guidelines.

Environmental barriers probably include limited availability of pulmonary function testing in specialty clinics as well as physical or financial access to pulmonologists and respiratory therapists trained in neuromuscular diseases. In 2000, in a survey of Muscular Dystrophy Association clinic directors, Bach and Chaudhry ${ }^{29}$ found that $50 \%$ of clinics routinely perform pulmonary function tests, $34 \%$ polysomnograms, $25 \%$ sleep oximetry, $15 \%$ daytime oximetry, and only $3 \%$ daytime and/or sleep capnography. Likewise, we informally surveyed MD STARnet sites in 2009 and found that most sites did not perform pulmonary function testing in the neuromuscular or Muscular Dystrophy Association sponsored clinics (data not shown).
The ATS recommendations include twice yearly visits to a physician specializing in neuromuscular care after age 12 y or full-time wheelchair use; once an individual requires the use of mechanically assisted ventilation, a minimum of 2 visits to a pulmonologist per year is recommended. In a formal interview of caregivers of individuals with DMD in MD STARnet (interviews between April 2007 and March 2012), only 25\% of individuals over age 12 y or wheelchair users who were not using mechanically assisted ventilation saw a pulmonologist semiannually. Of those individuals who were using mechanically assisted ventilation, $54 \%$ had a minimum of 2 visits/y. ${ }^{30}$

A paucity of pediatric and adult pulmonologists with specific expertise in neuromuscular respiratory medicine might preclude adherence to the twice yearly visit guideline in many centers, as it probably did in some of the MD STARnet sites. There is also probably a lack of infrastructure for transition of care from pediatric to adult respiratory care providers. Last, reimbursement for durable medical equipment (eg, noninvasive respiratory care devices) is dynamic, continues to be variable, and can be a major barrier for individuals and families with private insurance. ${ }^{31}$

Broad educational campaigns by national, state, and local agencies are needed to increase awareness and understanding of the guidelines and improve adherence. We need to train all health-care providers who potentially deliver respiratory care, including pediatric and adult neurologists, physiatrists, pulmonologists, intensivists, respiratory therapists, and emergency room and primary care physicians. It is imperative to convey that the decline in lung function in DMD is silent, and regular monitoring of lung function can identify individuals at risk of acute respiratory failure. Only then can respiratory assist devices be prescribed proactively instead of reactively during or after a hospital admission.

Broader access to pulmonary function testing within clinics is needed and should include key testing, such as spirometry, cough peak flow, pulse oximetry, and measures of body $\mathrm{CO}_{2}$ by capnography $\mathrm{P}_{\mathrm{ETCO}_{2}}$ or blood or transcutaneous $\mathrm{P}_{\mathrm{CO}_{2}}$. A respiratory therapist in the neuromuscular clinic or in a pulmonary function test laboratory can provide this testing.

Patients in respiratory failure in the intensive care unit also need a health-care provider who can advocate for noninvasive modalities and extubation protocols. Bach et $\mathrm{al}^{32}$ reported a first-attempt protocol extubation success rate of $95 \%$ for 149 subjects with severe respiratory muscle weakness from neuromuscular disease. This protocol included aggressive airway clearance with mechanical inexsufflation while intubated and extubating to NIV when the individual had weaned to room air, had a clear chest radiograph and minimal secretions, and was off respiratory depressants. Clearly, extubation protocols can be success- 


\section{Respiratory Care Received in DMD}

ful for many individuals with DMD, but they require a culture shift away from the premature use of tracheostomies to achieve patient extubation.

\section{Conclusions}

In a large sample of adolescents and adults affected by DMD between 2000 and 2010, monitoring of respiratory function and use of respiratory assist devices occurred less often than recommended by the 2004 ATS guidelines. Additional investigations are needed to elucidate the causes of non-adherence and potential solutions. Encouragement of partnerships between all physicians treating patients with DMD and neuromuscular respiratory specialists is warranted to ensure patient access to the respiratory interventions that have been shown to improve survival and quality of life.

\section{ACKNOWLEDGMENTS}

We acknowledge the efforts of the study coordinators, interviewers, and data manager in data collection and cleaning.

\section{REFERENCES}

1. Romitti PA, Zhu Y, Puzhankara S, James KA, Nabukera SK, Zamba GKD, et al. Prevalence of Duchenne and Becker muscular dystrophies in the United States. Pediatrics 2015;135(3):513-521.

2. Bach JR, Alba AS. Management of chronic alveolar hypoventilation by nasal ventilation. Chest 1990;97(1):52-57.

3. Gozal D. Pulmonary manifestations of neuromuscular disease with special reference to Duchenne muscular dystrophy and spinal muscular atrophy. Pediatr Pulmonol 2000;29(2):141-150.

4. Finder JD, Birnkrant D, Carl J, Farber HJ, Gozal D, Iannaccone ST, et al. Respiratory care of the patient with Duchenne muscular dystrophy: ATS consensus statement. Am J Respir Crit Care Med 2004; 170(4):456-465.

5. Humbertclaude V, Hamroun D, Bezzou K, Bérard C, BoespflugTanguy $\mathrm{O}$, Bommelaer $\mathrm{C}$, et al. Motor and respiratory heterogeneity in Duchenne patients: implication for clinical trials. Eur J Paediatr Neurol 2012;16(2):149-160.

6. Benditt JO, Boitano L. Respiratory support of individuals with Duchenne muscular dystrophy: toward a standard of care. Phys Med Rehabil Clin N Am 2005;16(4):1125-1139, xii.

7. Boitano LJ. Management of airway clearance in neuromuscular disease. Respir Care 2006;51(8):913-922; discussion 922-924.

8. Eagle M, Bourke J, Bullock R, Gibson M, Mehta J, Giddings D, et al. Managing Duchenne muscular dystrophy: the additive effect of spinal surgery and home nocturnal ventilation in improving survival. Neuromuscul Disord 2007;17(6):470-475.

9. Ishikawa Y, Miura T, Ishikawa Y, Aoyagi T, Ogata H, Hamada S, Minami R. Duchenne muscular dystrophy: survival by cardio-respiratory interventions. Neuromuscul Disord 2011;21(1):47-51.

10. Bach JR, Ishikawa Y, Kim H. Prevention of pulmonary morbidity for patients with Duchenne muscular dystrophy. Chest 1997;112(4): 1024-1028.

11. Gomez-Merino E, Bach JR. Duchenne muscular dystrophy: prolongation of life by noninvasive ventilation and mechanically assisted coughing. Am J Phys Med Rehabil 2002;81(6):411-415.

12. Jeppesen J, Green A, Steffensen BF, Rahbek J. The Duchenne muscular dystrophy population in Denmark, 1977-2001: prevalence, in- cidence and survival in relation to the introduction of ventilator use. Neuromuscul Disord 2003;13(10):804-812.

13. Simonds AK. Recent advances in respiratory care for neuromuscular disease. Chest 2006;130(6):1879-1886.

14. Toussaint M, Chatwin M, Soudon P. Mechanical ventilation in Duchenne patients with chronic respiratory insufficiency: clinical implications of 20 years published experience. Chron Respir Dis 2007; 4(3):167-177.

15. Toussaint M, Soudon P, Kinnear W. Effect of non-invasive ventilation on respiratory muscle loading and endurance in patients with Duchenne muscular dystrophy. Thorax 2008;63(5):430-434.

16. Birnkrant DJ, Bushby KM, Amin RS, Bach JR, Benditt JO, Eagle M, et al. The respiratory management of patients with Duchenne muscular dystrophy: a DMD Care Considerations Working Group specialty article. Pediatr Pulmonol 2010;45(8):739-748.

17. Bushby K, Finkel R, Birnkrant DJ, Case LE, Clemens PR, Cripe L, et al. Diagnosis and management of Duchenne muscular dystrophy, part 1: diagnosis, and pharmacological and psychosocial management. Lancet Neurol 2010;9(1):77-93.

18. Bushby K, Finkel R, Birnkrant DJ, Case LE, Clemens PR, Cripe L, et al. Diagnosis and management of Duchenne muscular dystrophy, part 2: implementation of multidisciplinary care. Lancet Neurol 2010; 9(2):177-189.

19. Miller LA, Romitti PA, Cunniff C, Druschel C, Mathews KD, Meaney FJ, et al. The Muscular Dystrophy Surveillance Tracking and Research Network (MD STARnet): surveillance methodology. Birth Defects Res A Clin Mol Teratol 2006;76(11):793-797.

20. Mathews KD, Cunniff C, Kantamneni JR, Ciafaloni E, Miller T, Matthews D, et al. Muscular Dystrophy Surveillance Tracking and Research Network (MD STARnet): case definition in surveillance for childhood-onset Duchenne/Becker muscular dystrophy. J Child Neurol 2010;25(9):1098-1102.

21. Imbornoni L, Price ET, Andrews J, Meaney FJ, Ciafaloni E, Cunniff C. Diagnostic and clinical characteristics of early-manifesting females with Duchenne or Becker muscular dystrophy. Am J Med Genet A 2014;164A(11):2769-2774.

22. Kim S, Campbell KA, Fox DJ, Matthews DJ, Valdez R. Corticosteroid treatments in males with Duchenne muscular dystrophy: treatment duration and time to loss of ambulation. J Child Neurol 2015; 30(10):1275-1280

23. Fox DJ, Kumar A, West NA, DiRienzo AG, James KA, Oleszek J, Muscular Dystrophy Surveillance, Tracking, and Research Network (MD STARnet). Trends with corticosteroid use in males with Duchenne muscular dystrophy born 1982-2001. J Child Neurol 2015; 30(1):21-26.

24. Eagle M, Baudouin SV, Chandler C, Giddings DR, Bullock R, Bushby K. Survival in Duchenne muscular dystrophy: improvements in life expectancy since 1967 and the impact of home nocturnal ventilation. Neuromuscul Disord 2002;12(10):926-929.

25. Katz SL, McKim D, Hoey L, Barrowman N, Kherani T, Kovesi T, et al. Respiratory management strategies for Duchenne muscular dystrophy: practice variation amongst Canadian subspecialists. Pediatr Pulmonol 2013;48(1):59-66.

26. Rodger S, Woods KL, Bladen CL, Stringer A, Vry J, Gramsch K, et al. Adult care for Duchenne muscular dystrophy in the UK. J Neurol 2015;262(3):629-641.

27. Francke AL, Smit MC, de Veer AJ, Mistiaen P. Factors influencing the implementation of clinical guidelines for health care professionals: a systematic meta-review. BMC Med Inform Decis Mak 2008; 8:38-49.

28. Cabana MD, Rand CS, Powe NR, Wu AW, Wilson MH, Abboud PA, Rubin HR. Why don't physicians follow clinical practice guidelines: a framework for improvement. JAMA 1999;282(15):14581465. 


\section{Respiratory Care Received in DMD}

29. Bach JR, Chaudhry SS. Standards of care in MDA clinics: Muscular Dystrophy Association. Am J Phys Med Rehabil 2000;79(2):193196.

30. Pandya SK, Campbell KA, Andrews JG, Meaney FJ, Ciafaloni E. Health services received by individuals with Duchenne/Becker muscular dystrophy. Muscle Nerve 2016;53(2):191-197
31. Finder JD. A 2009 perspective on the 2004 American Thoracic Society Statement, "respiratory care of the patient with Duchenne muscular dystrophy." Pediatrics 2009;123(Suppl 4):S239-S241.

32. Bach JR, Gonçalves MR, Hamdani I, Winck JC. Extubation of patients with neuromuscular weakness: a new management paradigm. Chest 2010;137(5):1033-1039. 